\title{
Prevalence of Traumatic Brain Injuries and Comparison of Its Clinical Findings from Mild to Severe Grade on Computed Tomography
}

\author{
Ayesha Umar ${ }^{1 *}$, Akash John ${ }^{2}$, Muhammad Ahmad Naeem ${ }^{3}$, Hussnain Rashid Butt ${ }^{4}$, Abid Ali $^{5}$ \\ ${ }^{14}{ }^{4}$ Medical Imaging Doctor, Department of Radiological Sciences and Medical Imaging, University of Lahore, Gujrat, Pakistan \\ ${ }^{2}, 3$ Lecturer, Department of Radiological Sciences and Medical Imaging, University of Lahore, Gujrat, Pakistan \\ ${ }^{5}$ Associate Professor, Department of Allied Health Sciences, University of Lahore, Gujrat, Pakistan
}

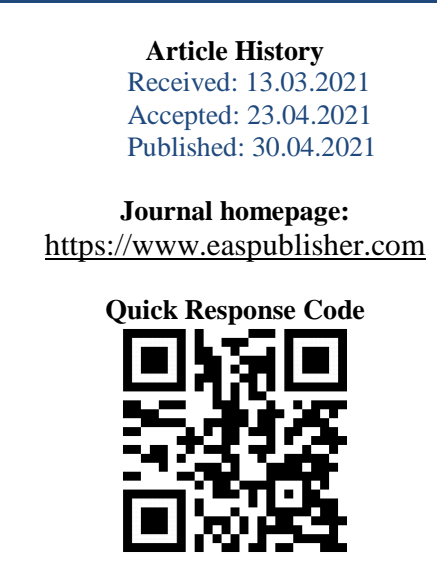

Abstract: Background: Traumatic brain injury and its implications lead to huge public health issues. Annually, TBI affects approximately 50 million individuals all around the world. Head injury is the leading neurological reason for despondency and fatality, particularly influencing the youngsters. $85 \%$ to $90 \%$ are mild or moderate TBI. Objective: To find prevalence of traumatic brain injuries on computed tomography and that of severity classification from mild to severe grade. Material and Methods: 100 patients with history of head trauma were evaluated in this study, which came to emergency department of Public sector. GCS was taken and all were referred to CT. Noncontrast CT of brain was performed with slice thickness of $3 \mathrm{~mm}$ from skull base to vertex. And analysis was done using (SPSS) Statistical Package for the Social Sciences version 21. Results: Of 100 TBI patients, men composed $66 \%$ and women $34 \%$. Falls (38\%), violence (2\%) and other causes $(5 \%)$ all collectively cause fewer traumas than RTA (55\%) specifically in patients of age group 16-26 years. Different traumatic brain injuries were noted including EDH (22\%), IPH (23\%), SAH (9\%), SDH (27\%), Contusion (4\%) and infarction (15\%). Noteworthy, most of the brain injuries were observed as mild TBI in $65 \%$ of cases accounting for more than moderate $(21 \%)$ and severe TBI (14\%). Conclusion: The study concludes that $65 \%$ of patients who acquired head trauma had mild TBI while remaining $21 \%$ and $14 \%$ consumed moderate and severe TBI respectively. Subdural and intraparenchymal hemorrhages are most frequent type of diagnosis in TBI.

Keywords: Traumatic brain injury, Computed Tomography, Subdural hemorrhage, Epidural hemorrhage, Intra-parenchymal hemorrhage, subarachnoid hemorrhage, Road Traffic Accident. Abbreviations: TBI: Traumatic Brain Injury; CT: Computed Tomography; GCS: Glasgow Coma Scale; EDH: Epidural Hemorrhage; IPH: Intraparenchymal Hemorrhage; SDH: Subdural Hemorrhage; SAH: Subarachnoid Hemorrhage; RTA: Road Traffic Accidents

Copyright () 2021 The Author(s): This is an open-access article distributed under the terms of the Creative Commons Attribution 4.0 International License (CC BY-NC 4.0) which permits unrestricted use, distribution, and reproduction in any medium for non-commercial use provided the original author and source are credited.

\section{INTRODUCTION}

Traumatic brain injury (TBI) could be specified as a brain activity disturbance arising from an immediate, unpredictable, unsustainable physical force applied [1]. Falls are the second major source of TBI with $38 \%$ as compared to road accidents $(51 \%)$ which is still ranked at top [2] Excluding the adolescents whose mortality occurs because of abuse, in $80 \%$ of patients [3]. On the basis of neuropathology, there are two types of TBI (a) focal damage of brain, caused by contact type of injury that consequently leads to cerebral laceration, brain hemorrhage and cerebral contusion (b) diffuse damage of brain, caused by acceleration and deceleration force that leads to bruising of brain or diffuse axonal shearing [4]. All brain injuries are not primary that appear quickly at the time of impact but some are secondary brain injuries that develop gradually[5].

Traumatic brain injury varies in case of sign and symptoms, pathophysiology, and outcome along with mortality rate reaching less than $1 \%$ in case of mild TBI and about $40 \%$ in case of severe TBI [2]. Out of total TBI $80-90 \%$ are mild TBI [6].

Based on three indicators: Post Traumatic Amnesia (PTA), Glasgow Coma Scale (GCS), and loss of consciousness, traumatic brain injury is classified as mild, moderate, and severe. Severe TBI has GCS of 3 to 8 , coma duration greater than $36 \mathrm{hr}$, and PTA of more than a week. The GCS ranges from 9 to 12 for moderate TBI, coma duration is from $20 \mathrm{~min}-36 \mathrm{hr}$ and has PTA 
of 1 to 7 days [7]. Patients of 13 to 15 Glasgow Coma Scale are considered to have mild injury [8] with coma duration of 30 minutes and PTA of about 24 hours [9].

TBI most often contributes to diffuse axonal injury, where temporal lobe and frontal lobe are more susceptible cortical areas. Contusion, cerebral oedema, brain ischemia, and intracerebral hemorrhages may result from head injury [7].

Diffuse axonal injury (DAI) is a form of injury in which the brain axons shears. DAI typically occurs due to the motion of acceleration/deceleration that induces white matter tract shearing. DAI usually injures white matter tracts of corpus callosum as well as of brainstem. Ten percent of TBI cases are diagnosed with some extend of diffuse axonal injury $[10,11]$.

Cortical contusion commonly occurs when the skull's rough internal surface injures the brain during the impact. The coup contusions are commonly more intense as compare to countercoup site [12]. In computed tomography, it is normally seen as hemorrhagic lesion. A portion of contusion may be seen as hypodense or as isodensity [13].

Brain injury can lead to intracerebral hemorrhage by direct or indirect trauma to blood vessels deep within the skull, and such harms may contribute to intracerebral hemorrhage outside or inside the parenchyma of the brain. Frequently occurring forms of ICH include (a) subarachnoid hemorrhage emerges as injury take place in artery or veins passing via the subarachnoid cavity surrounding the brain and diagnosed on CT scan as irregular hyperdense areas in subarachnoid cavity [14]. While its clinical features are considerably distinct in several cases from either the appearance of cerebral ischemia or acute ICH [15]. (b) Epidural hemorrhage grows a biconvex mass in between the outer most layers of brain (dura matter) and skull resulting in their separation. Most often occur by the rupture of middle meningeal artery. It appears as well-defined, oval shaped hyper-dense mass in epidural space. (c) Subdural hemorrhage normally occurs by the disruption of bridging veins through abrupt deceleration of head. Acute subdural hematoma is diagnosed on CT as uniform, hyper-dense, concave mass in subdural cavity [14].

Nearly all head injuries will potentially progress to brain ischemia that can cause fatality if left unchecked. The advancement in MDCT has affectively lowered the scan time and permits rapid reimaging of the slices which are blurred via blur artifact [16]. Brain ischemia is identified on computed tomography during early hours as there is no Gray-White differentiation due to rise in fluid level of ischemic cells [17]. CT is the modality of choice for identifying vast range of disorders and traumas. The significance of CT scan in treatment is that it permits the physicians to distinguish among [7, 3] cerebral hemorrhage, cerebral ischemia, concussion, and stroke [18].

TBI and its implications are producing substantial social and economic burden and considered third major source of mortality globally. There is necessity of more research work about this subject. This study will address the prevalence of all types of TBI ranging from mild to severe and significance of Computed tomography (that is much affordable and accessible, has short scanning period and it makes easy to scan the individual with supplemental oxygen, traction or anxiety) is the first-line modality within 24 hours of trauma. This study results will be helpful in planning public health measures, prevention policies and rehabilitation measures in desire to lower the dramatically increasing burden of TBI in the society.

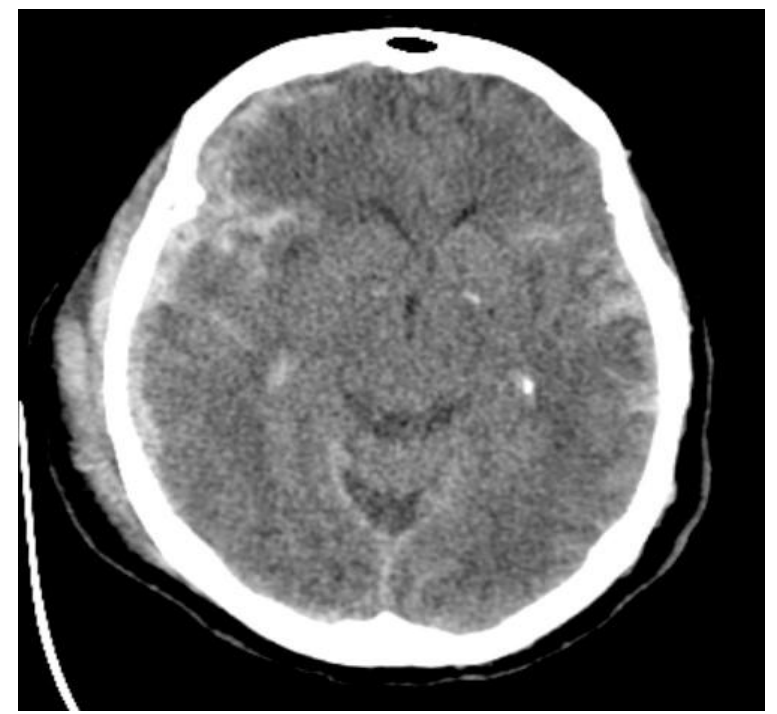

Fig-1: A patient with history of trauma representing acute subarachnoid hemorrhage

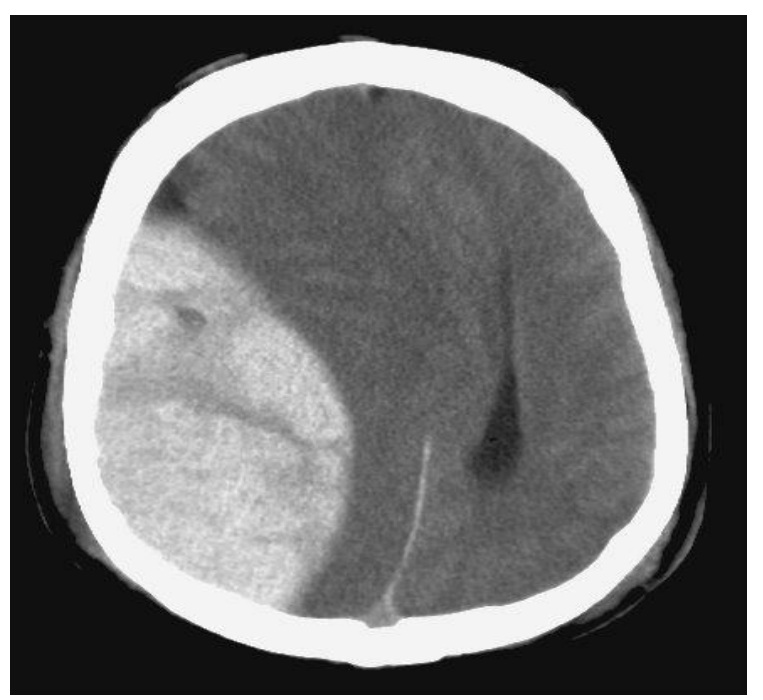

Fig-2: Image depicting typical acute epidural hemorrhage in right hemisphere of brain, what should be observed is concave mass causing massive mass effect on mid line 


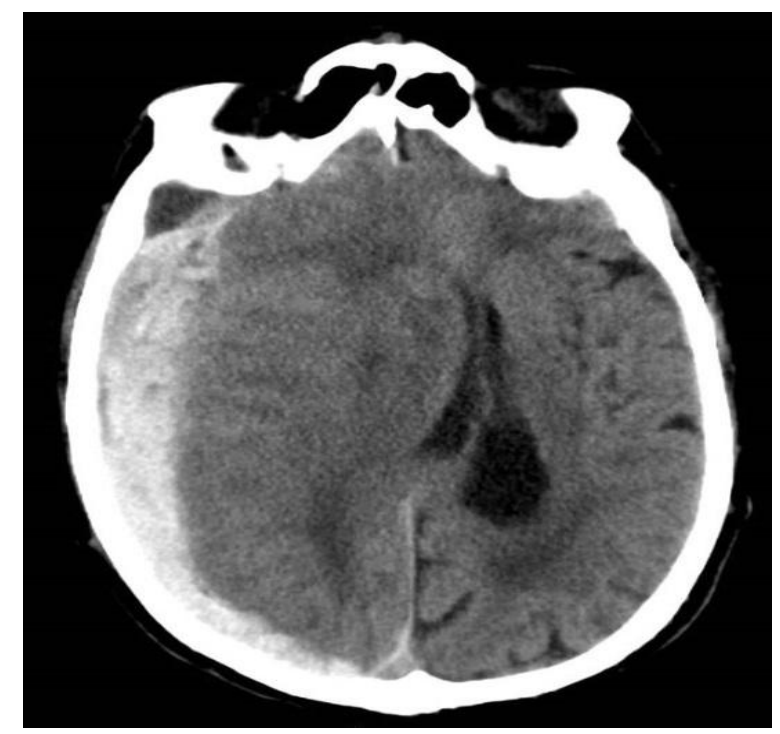

Fig-3: Image showing acute subdural hemorrhage in right hemisphere on noncontract CT, giving appearance of homogeneous hyper dense, crescenteric mass causing mass effect on falx cerebri

\section{Materials ANd Methods}

This study was conducted in Department of radiology at public sector hospital. We deliberated on different types of pathologies after evaluating head trauma on computed tomography including hemorrhage, strokes, diffuse axonal injury, concussions, and edema. Between August 2020 and February 2021, we diagnosed multiple cases of traumatic brain injuries along with Glasgow Coma Scale as qualifier to define their eligibility for severity classification of TBI. Almost 100 patients of above-mentioned pathologies were studied for research work in a given period. Demographics including age, gender, history, and mechanism of trauma. These types of pathologies were confirmed by computed tomography scan.

\section{Inclusion Criteria}

All the patients of age ranging between 16 to 60 years, who came in CT department with a history of head trauma

\section{Exclusion Criteria}

Patients with brain tumor, bleeding disorder, anticoagulant therapy, Cerebrovascular attack, Cerebral palsy, and patients below 16 and above 60 years of age.

\section{Measuring tool}

We used Glasgow Coma Scale as a tool to measure the severity of traumatic brain injury. GCS was calculated on the basis of three behaviors such as eyeopening containing 4 points, verbal response having 5 points and motor response consisting of 6 points. The patient was scored based on his condition and obtained scores are then added up to give a final value of GCS.

All head trauma patients were evaluated by CT scan. All patients' scan was conducted on CT scanner of 64 slice Toshiba Aquilion. Images were obtained using slice thickness of $3 \mathrm{~mm}$ from foramen magnum to vertex of head. Noncontrast CT images were obtained because of the indications. Using $120 \mathrm{kvp}, 300 \mathrm{~mA}$ tube current and orbitometal line as reference point the helical scan of brain was completed in about 4 to $5 \mathrm{sec}$. Axial images obtained were then evaluated by the radiologist in the viewing console to give diagnosis. In acute phase of head injury, CT is the frequently used imaging modality due to more precise diagnosis of subarachnoid and parenchymal hemorrhage. Data entry, processing and analysis were made using (SPSS) Statistical Package for the Social Sciences version 21.

\section{RESULTS}

Total 100 TBI patients were evaluated between December 2019 and May 2020. Table 1 shows statistics with mean age 35.9 , minimum age of 16 and maximum age of 60 and standard deviation of 16.0, Table 2 shows gender distribution in which $66 \%$ were males and $34 \%$ were females who acquired brain trauma. Out of all determined etiologies, RTA was most frequent injury mechanism. The cause of head trauma was RTA in $55 \%$, fall in $38 \%$, violence in $2 \%$ and other causes in $5 \%$ of cases as illustrated in table 3 . Whereas able 4 depicts that out of $100 \mathrm{TBI}$ patients, 04(4\%) represented brain contusion, $15(15 \%)$ represented infarction and $81(81 \%)$ represented different types of brain hemorrhage. Out of $81,22(27 \%)$ showed epidural hemorrhage (EDH), 23(28\%) showed intraparenchymal hemorrhage (IPH), 9(11\%) showed subarachnoid hemorrhage (SAH) and 27(33\%) showed subdural hemorrhage (SDH). Severity classification of TBI on the basis of GCS, in which out of these 100 patients, $65 \%$ were consuming mild TBI, $21 \%$ were diagnosed with moderate TBI and $14 \%$ were representing severe TBI as portrayed in table 5 .

Table-1: Descriptive Statistics

\begin{tabular}{|l|l|l|l|l|l|}
\hline & $\mathbf{N}$ & Minimum & Maximum & Mean & Std. Deviation \\
\hline Age & 100 & 16.0 & 60.0 & 35.9 & 16.0 \\
\hline
\end{tabular}

Table-2: Gender distribution of participants

\begin{tabular}{|l|l|l|l|l|}
\hline & Frequency & Percent & Valid Percent & Cumulative Percent \\
\hline Male & 66 & 66.0 & 66.0 & 66.0 \\
\hline Female & 34 & 34.0 & 34.0 & 100.0 \\
\hline Total & 100 & 100.0 & 100.0 & \\
\hline
\end{tabular}




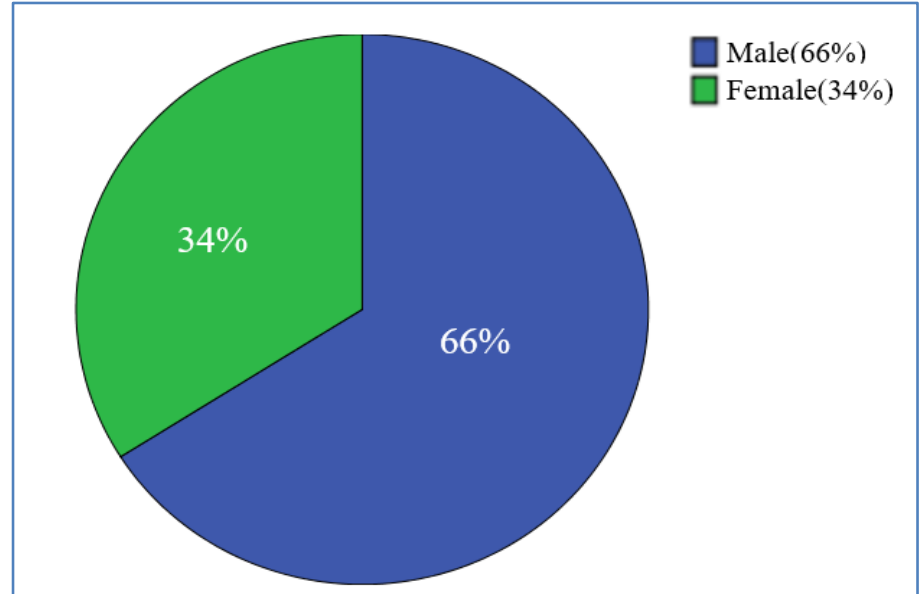

Fig-4: Gender distribution of all TBI patients

Table-3: All possible causes of traumatic brain injuries

\begin{tabular}{|l|l|l|l|l|}
\hline & Frequency & Percent & Valid Percent & Cumulative Percent \\
\hline RTA & 55 & 55.0 & 55.0 & 55.0 \\
\hline Fall & 38 & 38.0 & 38.0 & 93.0 \\
\hline Violence & 2 & 2.0 & 2.0 & 95.0 \\
\hline other causes & 5 & 5.0 & 5.0 & 100.0 \\
\hline Total & 100 & 100.0 & 100.0 & \\
\hline
\end{tabular}

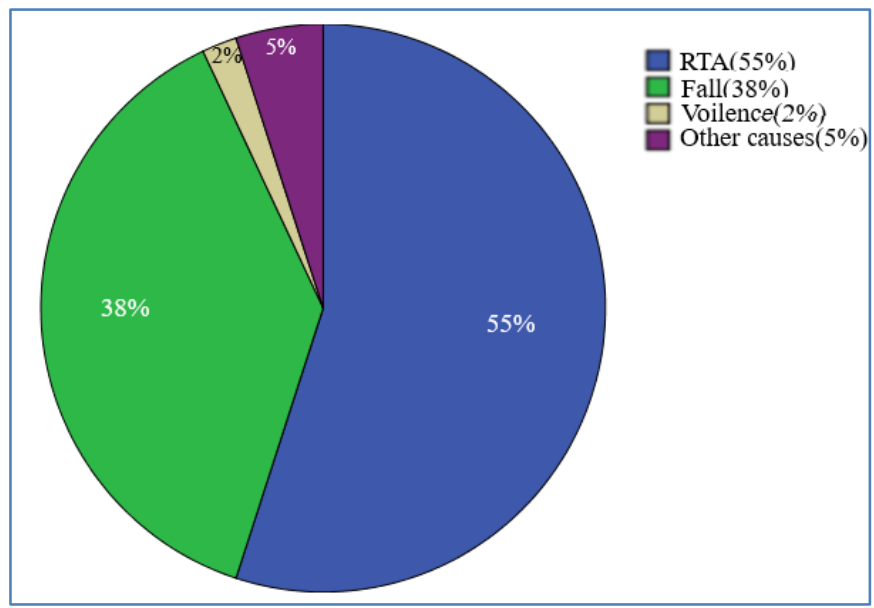

Fig-5: Showing occurrence of different injury mechanisms of TBI

Table-4: Prevalence of diagnosed traumatic brain injuries in this study

\begin{tabular}{|l|l|l|l|l|}
\hline & Frequency & Percent & Valid Percent & Cumulative Percent \\
\hline Epidural hemorrhage & 22 & 22.0 & 22.0 & 22.0 \\
\hline Intra Parenchymal hemorrhage & 23 & 23.0 & 23.0 & 45.0 \\
\hline Subarachnoid hemorrhage & 9 & 9.0 & 9.0 & 54.0 \\
\hline subdural hemorrhage & 27 & 27.0 & 27.0 & 81.0 \\
\hline Infarction & 15 & 15.0 & 15.0 & 96.0 \\
\hline Contusion & 4 & 4.0 & 4.0 & 100.0 \\
\hline Total & 100 & 100.0 & 100.0 & \\
\hline
\end{tabular}




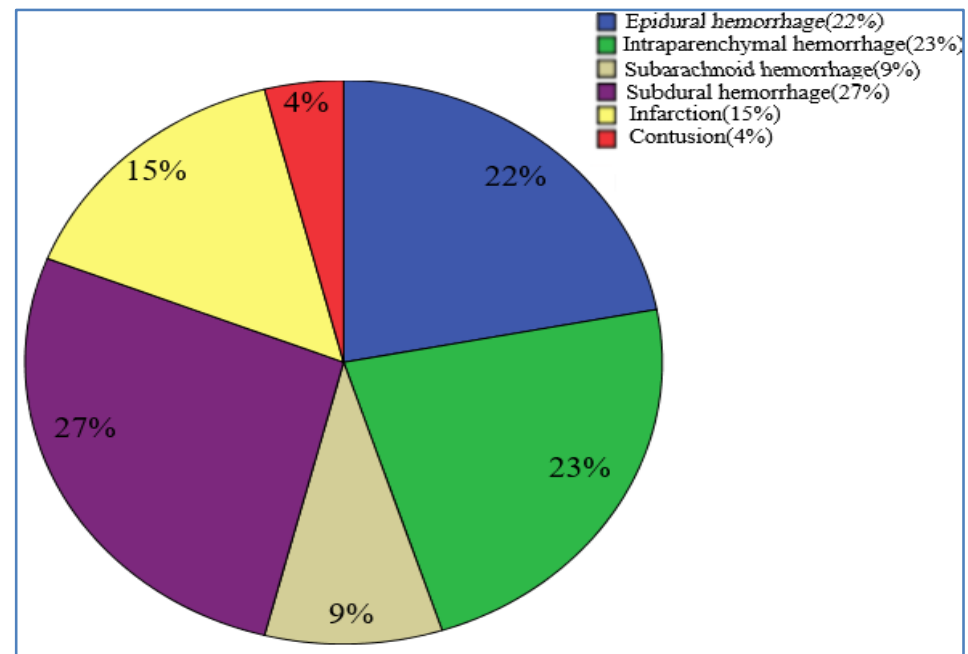

Fig-5: Showing occurrence of different injury mechanisms of TBI

Table-5: Prevalence of mild to severe TBI

\begin{tabular}{|l|l|l|l|l|}
\hline & Frequency & Percent & Valid Percent & Cumulative Percent \\
\hline Mild & 65 & 65.0 & 65.0 & 65.0 \\
\hline Moderate & 21 & 21.0 & 21.0 & 86.0 \\
\hline Severe & 14 & 14.0 & 14.0 & 100.0 \\
\hline Total & 100 & 100.0 & 100.0 & \\
\hline
\end{tabular}

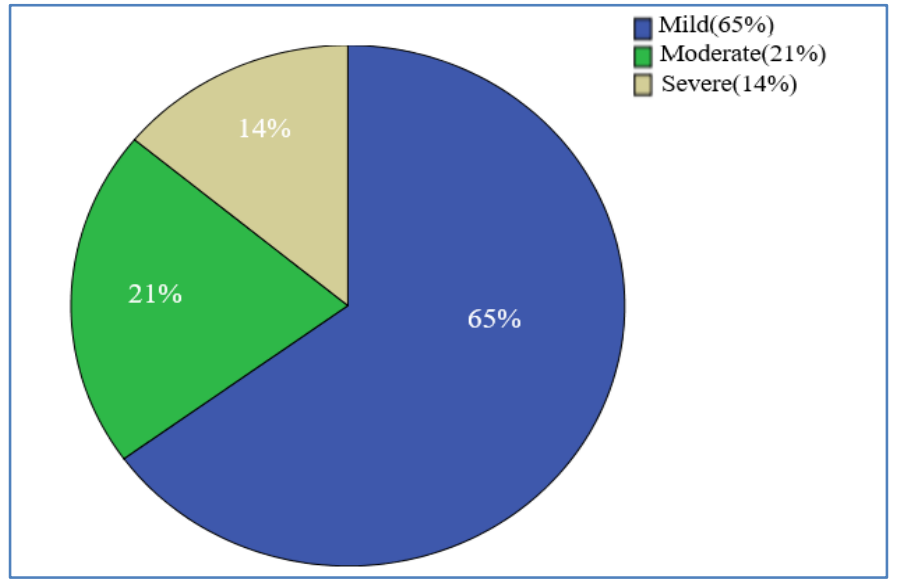

Fig-7: Severity classification of TBI and its prevalence

\section{DiscuSSION}

TBI is taken into account as really worrying socio-economic health problem and foretold to surpass several diseases as a significant explanation for death and incapacity for year 2020. Computed Tomography has vital role both in evaluation and treatment of TBI, especially it is significant for triage in critical setups in deciding which patient needs immediate neurological treatment. The leading external source of head injury and subsequently TBI are Road Traffic Accidents (in approximately $60 \%$ of cases), workplace and sportsrelated activities $(10 \%)$, violence $(10 \%)$ and falls 20 $30 \%$ [19].

The estimated crude annual incidence of mild traumatic brain injury was 224/100000, moderate was $23 / 100000$ and severe traumatic brain injury was
$13 / 100000$ regarding to the meta-analysis and systematic review study of TBI carried on Medline and EMBASE by May 23, 2014 [20].

This study intended to evaluate the prevalence of different traumatic brain injuries using computed tomography and prevalence of TBI from mild to severe grade using Glasgow coma scale in group of patients with recent history of head trauma. Mostly TBI happened in patients of active age and affected men more than women. RTA as mechanism of injury was predominantly accountable for TBI. This study indicated that the occurrence of mild traumatic brain injury is maximum with 65 percent than moderate or severe and subdural hemorrhage is the most frequent traumatic brain injury that affects the brain. 
In several aspects, the results of this research are similar to those of other traumatic brain injury epidemiological studies, especially when it comes to demographic variables including age, sex, and injury mechanism as discussed below.

Research conducted in Australia included 413 confirmed cases of TBI. Though majority of the cases (257) were mild (62\%), 84 cases were having moderate injury $(20.3 \%)$ and 56 cases were of severe TBI $(13.6 \%)$. In youngsters and old age groups the common injury event was falls while in adults the frequent mechanism of injury was road traffic accidents [21].

Muhammad Ali Subhani et al. conducted research on 100 head trauma patients in which $31 \%$ were females and $69 \%$ were males and gave results according to which $31.6 \%$ showed subdural hemorrhage $(\mathrm{SDH}), 10.5 \%$ represented subarachnoid hemorrhage (SAH), 26.3\% showed epidural hemorrhage (EDH) and $31.6 \%$ represented intraparenchymal hemorrhage (IPH). Out of these 100 cases, contusion and infarction account for $4 \%$ and $25 \%$ respectively [19]. His results closely resemble this study results.

Teuntje M.J.C. Andriessen at el performed a study on 508 TBI patients. According to his study, the mechanism of injury was RTA in $51 \%$ cases and fall in about $38 \%$ of cases but did not discuss the frequency of violence and other causes [2]. His results are almost similar to this research except we also rule out the occurrence of violence and other causes were $2 \%$ and $5 \%$ respectively.

\section{CONCLUSION}

This study concludes that $66 \%$ of patients who acquired head trauma had mild TBI while remaining $21 \%$ and $14 \%$ consumed moderate and severe TBI respectively. Subdural and intraparenchymal hemorrhage are most frequent type of diagnosis in TBI.

\section{Conflict of Interest}

Any conflict of interest does not exist in this study.

\section{Funding}

This study does not have any funding or financial support.

\section{REFERENCES}

1. Bhatti, J. A., Stevens, K., Mir, M. U., Hyder, A. A., \& Razzak, J. A. (2015). Emergency care of traumatic brain injuries in Pakistan: a multicenter study. BMC emergency medicine, 15(2), 1-7.

2. Andriessen, T. M., Horn, J., Franschman, G., van der Naalt, J., Haitsma, I., Jacobs, B., ... \& Vos, P. E. (2011). Epidemiology, severity classification, and outcome of moderate and severe traumatic brain injury: a prospective multicenter study. Journal of neurotrauma, 28(10), 2019-2031.

3. Brunelle, F., \& Boddaert, N. (2005). Imagerie des traumatismes crâniens chez l'enfant. Journal de Radiologie, 86(2), 253-262.

4. Werner, C., \& Engelhard, K. (2007). Pathophysiology of traumatic brain injury. BJA: British Journal of Anaesthesia, 99(1), 4-9.

5. Ghajar, J. (2000). Traumatic brain injury. The Lancet, 356(9233):923-9.

6. Angerpointner, T. A. (2014). Computed tomographic scan in head trauma: What is the rational in children?. Journal of Pediatric Surgery, 49(7), 1181.

7. Vakil, E. (2005). The effect of moderate to severe traumatic brain injury (TBI) on different aspects of memory: a selective review. Journal of clinical and experimental neuropsychology, 27(8), 977-1021.

8. Van der Naalt, J., Van Zomeren, A. H., Sluiter, W. J., \& Minderhoud, J. M. (1999). One year outcome in mild to moderate head injury: the predictive value of acute injury characteristics related to complaints and return to work. Journal of Neurology, Neurosurgery \& Psychiatry, 66(2), 207-213.

9. Ponsford, J., Willmott, C., Rothwell, A., Cameron, P., Kelly, A. M., Nelms, R., ... \& Ng, K. I. M. (2000). Factors influencing outcome following mild traumatic brain injury in adults. Journal of the International Neuropsychological Society, 6(5), 568-579.

10. Gennarelli, T. A., Thibault, L. E., Adams, J. H., Graham, D. I., Thompson, C. J., \& Marcincin, R. P. (1982). Diffuse axonal injury and traumatic coma in the primate. Annals of Neurology: Official Journal of the American Neurological Association and the Child Neurology Society, 12(6), 564-574.

11. Vieira, R. D. C. A., Paiva, W. S., de Oliveira, D. V., Teixeira, M. J., de Andrade, A. F., \& Sousa, R. M. C. D. (2016). Diffuse axonal injury: epidemiology, outcome and associated risk factors. Frontiers in neurology, 7, 178.

12. Kim, J. J., \& Gean, A. D. (2011). Imaging for the diagnosis and management of traumatic brain injury. Neurotherapeutics, 8(1), 39-53.

13. Kurland, D., Hong, C., Aarabi, B., Gerzanich, V., \& Simard, J. M. (2012). Hemorrhagic progression of a contusion after traumatic brain injury: a review. Journal of neurotrauma, 29(1), 19-31.

14. Kidwell, C. S., \& Wintermark, M. (2008). Imaging of intracranial haemorrhage. The Lancet Neurology, 7(3), 256-267.

15. Feng, H., Jin, Z., He, W., \& Zhao, X. (2018). Cerebral venous outflow participates in perihematomal edema after spontaneous intracerebral hemorrhage: A cross-sectional study. Medicine, 97(35).

16. Lee, B., \& Newberg, A. (2005). Neuroimaging in traumatic brain imaging. NeuroRx, 2(2), 372-383.

17. Latchaw, R. E., Alberts, M. J., Lev, M. H., Connors, J. J., Harbaugh, R. E., Higashida, R. T., ... \& 
Walters, B. (2009). Recommendations for imaging of acute ischemic stroke: a scientific statement from the American Heart Association. Stroke, 40(11), 3646-3678.

18. Cramer, A., Hecla, J., Wu, D., Lai, X., Boers, T., Yang, K., ... \& Gupta, R. (2018). Stationary computed tomography for space and other resource-constrained environments. Scientific reports, 8(1), 1-10.

19. Asare, H. K., Abdulai, A. B., Akorli, E., Aweligiba, S., \& Akorli, P. (2019). Computed Tomography Findings of Traumatic Brain Injury in Patients with Head Trauma Presenting at the Tamale Teaching
Hospital, Ghana. Journal of Radiology and Clinical Imaging, 2(1), 24-32.

20. Nguyen, R., Fiest, K. M., McChesney, J., Kwon, C. S., Jette, N., Frolkis, A. D., ... \& Gallagher, C. (2016). The international incidence of traumatic brain injury: a systematic review and metaanalysis. Canadian journal of neurological sciences, 43(6), 774-785.

21. Tate, R. L., McDonald, S., \& Lulham, J. M. (1998). Incidence of hospital- treated traumatic brain injury in an Australian community. Australian and New Zealand journal of public health, 22(4), 419423.

Cite This Article: Ayesha Umar et al (2021). Prevalence of Traumatic Brain Injuries and Comparison of Its Clinical Findings from Mild to Severe Grade on Computed Tomography. EAS J Radiol Imaging Technol, 3(2), 119-125. 